\title{
Un guide de présentation pour les études qualitatives
}

\author{
Correspondance : ccdr-rmtc@phac-aspc.gc.ca
}

Citation proposée : Un guide de présentation pour les études qualitatives. Relevé des maladies transmissibles au Canada 2016;42:198-9. https://doi.org/10.14745/ccdr.v42i09a02f

Les études qualitatives permettent la connaissance approfondie de phénomènes complexes. À la différence des études fondées sur des mesures, qui quantifient ce qui se déroule dans un environnement expérimental, les études qualitatives permettent souvent d'expliquer les comportements et les perceptions dans les circonstances réelles. Dans l'optique des maladies transmissibles, les études qualitatives peuvent permettre d'examiner pourquoi les individus optent pour un comportement à haut risque, et identifier les facteurs qui influencent leurs décisions. Par exemple, une étude qualitative peut s'intéresser aux raisons pour lesquelles les professionnels de la santé n'appliquent pas de mesures adéquates d'hygiène des mains, et examiner si les patients peuvent aider en leur rappelant de le faire. Les résultats peuvent être surprenants. Par exemple, une étude récente a établi que les patients hospitalisés d'un hôpital, les plus insatisfaits des soins qu'ils recevaient étaient également les moins susceptibles de demander aux professionnels de la santé s'ils s'étaient lavé les mains (1). De plus, l'étude a établi que la décision de ne pas poser la question était liée à la conscience qu'avait le patient du degré peu élevé de satisfaction du personnel.

La recherche qualitative analyse des données issues de l'observation directe, d'entretiens en profondeur à questions ouvertes et de documents écrits. Les analyses inductives mettent au jour des tendances et des thèmes qui génèrent des hypothèses et établissent les bases de futures recherches. Bien que les études qualitatives n'établissent pas de preuves généralisables, un bon rapport de recherche offre l'information nécessaire pour que les lecteurs puissent juger du potentiel d'application ou de transfert des résultats à leur propre contexte (2).

Il existe plusieurs critères à respecter quand vient le temps de rédiger un rapport de recherche qualitative (3-6). Le Relevé des maladies transmissibles au Canada (RMTC) les résume dans une liste de vérification en 24 points, qui inclut les lignes directrices COREQ (Consolidated criteria for reporting qualitative research) du réseau international EQUATOR (6). La liste du RMTC insiste sur l'importance de décrire comment les données ont été recueillies et résumées, quelles tendances ont été mises au jour, en explorent des résultats corroborants, offrant des explications alternatives et décrivant des prochaines étapes ou nouveaux champs de recherche sur lesquels elle ouvre (tableau 1).

Les rapports de recherche qualitative sont généralement d'environ 2500 mots - sans compter le résumé, les tableaux et les références. Comme pour toutes les soumissions, vérifier la section Renseignements à l'intention des auteurs du RMTC, publiée au début de chaque volume, chaque année en janvier, pour la préparation générale d'un article et les exigences de soumissions (7).

Tableau 1 : Liste de vérification pour les études qualitatives

\begin{tabular}{|c|c|c|}
\hline $\begin{array}{l}\text { Élément de la } \\
\text { déclaration }\end{array}$ & $\mathrm{N}^{\circ}$ & Description \\
\hline \multicolumn{3}{|l|}{ Titre/Résumé } \\
\hline Titre & 1 & $\begin{array}{l}\text { Donner un titre qui comprend le terme } \\
\text { "qualitatif », la population, la condition, le } \\
\text { lieu et le moment. }\end{array}$ \\
\hline Résumé & 2 & $\begin{array}{l}\text { Utiliser un format structuré pour le résumé } \\
\text { avec les en-têtes de section suivants: } \\
\text { contexte, objectif, méthodologie, résultats } \\
\text { et conclusion. }\end{array}$ \\
\hline \multicolumn{3}{|l|}{ Introduction } \\
\hline $\begin{array}{l}\text { Présentation du } \\
\text { sujet }\end{array}$ & 3 & $\begin{array}{l}\text { Présenter le sujet de l'étude et expliquer } \\
\text { pourquoi il est important. }\end{array}$ \\
\hline $\begin{array}{l}\text { Revue } \\
\text { documentaire }\end{array}$ & 4 & $\begin{array}{l}\text { Fournir un résumé de la littérature sur le } \\
\text { sujet et repérer toute lacune existante. }\end{array}$ \\
\hline $\begin{array}{l}\text { Justification de } \\
\text { l'étude }\end{array}$ & 5 & $\begin{array}{l}\text { Préciser la justification pour l'étude. La } \\
\text { justification pour l'utilisation de méthodes } \\
\text { qualitatives peut être précisée ici ou dans } \\
\text { la section Méthodologie. }\end{array}$ \\
\hline Objectif & 6 & Décrire clairement l'objectif de l'étude. \\
\hline $\begin{array}{l}\text { Approbation } \\
\text { éthique }\end{array}$ & 7 & $\begin{array}{l}\text { Préciser ici ou dans la section } \\
\text { Méthodologie si un examen du comité } \\
\text { d'éthique s'imposait et, le cas échéant, } \\
\text { où s'est fait l'examen et où a été obtenue } \\
\text { l'approbation. }\end{array}$ \\
\hline \multicolumn{3}{|l|}{ Méthodologie } \\
\hline Milieu & 8 & $\begin{array}{l}\text { Décrire l'environnement de l'étude et la } \\
\text { nature de la relation entre le chercheur et } \\
\text { les participants à l'étude (le cas échéant). }\end{array}$ \\
\hline Approche & 9 & $\begin{array}{l}\text { Préciser les méthodes qualitatives } \\
\text { retenues (par exemple des entretiens, } \\
\text { une observation participante, etc.), toute } \\
\text { base théorique qui pourrait s'appliquer } \\
\text { (par exemple, la théorie ancrée dans la } \\
\text { pratique) et la justification de ces choix. }\end{array}$ \\
\hline Populations & 10 & $\begin{array}{l}\text { Décrire les groupes parmi lesquels des } \\
\text { individus étaient invités à participer à } \\
\text { l'étude. }\end{array}$ \\
\hline Échantillonnage & 11 & $\begin{array}{l}\text { Préciser la stratégie d'échantillonnage } \\
\text { retenue pour l'étude (par exemple un } \\
\text { échantillonnage théorique ou encore une } \\
\text { méthode en boule de neige). }\end{array}$ \\
\hline
\end{tabular}




\begin{tabular}{|c|c|c|}
\hline $\begin{array}{l}\text { Collecte de } \\
\text { données }\end{array}$ & 12 & $\begin{array}{l}\text { Décrire comment les outils de collecte de } \\
\text { données ont été élaborés (par exemple } \\
\text { grâce à des essais pilotes des guides } \\
\text { d'entrevue) et comment les données ont } \\
\text { été enregistrées (par exemple de façon } \\
\text { audio, audiovisuelle ou dans un journal de } \\
\text { terrain). }\end{array}$ \\
\hline Analyse & 13 & $\begin{array}{l}\text { Préciser comment les données ont été } \\
\text { gérées et analysées, y compris l'utilisation } \\
\text { de tout système logiciel, et sur quelles } \\
\text { bases ont été établies la crédibilité et } \\
\text { la transférabilité de l'information (par } \\
\text { exemple par un retour aux participants, } \\
\text { une fiabilité interobservateur ou une } \\
\text { triangulation). }\end{array}$ \\
\hline Synthèse & 14 & $\begin{array}{l}\text { Décrire comment les résultats ont } \\
\text { été synthétisés (par exemple, quels } \\
\text { principes et quels choix ont orienté } \\
\text { la reconnaissance de tendances et la } \\
\text { définition de catégorie? Comment les } \\
\text { thèmes majeurs et mineurs ont-ils été } \\
\text { élaborés?). }\end{array}$ \\
\hline \multicolumn{3}{|l|}{ Observations } \\
\hline Échantillon & 15 & $\begin{array}{l}\text { Préciser la taille totale de l'échantillon et } \\
\text { le taux de non-participation. }\end{array}$ \\
\hline $\begin{array}{l}\text { Population, } \\
\text { moment et lieu }\end{array}$ & 16 & $\begin{array}{l}\text { Remettre les résultats en contexte, } \\
\text { c'est-à-dire en donnant suffisamment de } \\
\text { détails sur les circonstances et le contexte } \\
\text { pour donner une idée de la population, } \\
\text { du moment et du lieu (par exemple par un } \\
\text { usage approprié des citations). }\end{array}$ \\
\hline Analyse & 17 & $\begin{array}{l}\text { Présenter une analyse qui est crédible } \\
\text { et convaincante (à savoir que les thèmes } \\
\text { découlent logiquement des résultats; } \\
\text { les relations entre les données, les } \\
\text { modèles théoriques et les perspectives } \\
\text { sont décrites; les interprétations sont } \\
\text { perspicaces, etc.). }\end{array}$ \\
\hline Comparaisons & 18 & $\begin{array}{l}\text { Explorer les résultats corroborants (par } \\
\text { exemple, par triangulation) et tenir } \\
\text { compte des opinions contradictoires } \\
\text { ou divergentes (par exemple, les cas } \\
\text { négatifs). }\end{array}$ \\
\hline Synthèse & 19 & $\begin{array}{l}\text { Présenter les résultats d'une façon qui } \\
\text { permette de répondre clairement à la, ou } \\
\text { aux, question(s) de recherche. }\end{array}$ \\
\hline \multicolumn{3}{|l|}{ Discussion } \\
\hline $\begin{array}{l}\text { Résumé des } \\
\text { principales } \\
\text { conclusions }\end{array}$ & 20 & $\begin{array}{l}\text { Résumer les principales conclusions } \\
\text { et préciser en quoi les résultats sont } \\
\text { pertinents en regard des objectifs de } \\
\text { l'étude. }\end{array}$ \\
\hline Forces et faiblesses & 21 & $\begin{array}{l}\text { Préciser les forces et les faiblesses de } \\
\text { l'étude et examiner les explications } \\
\text { alternatives possibles des résultats } \\
\text { lorsqu'ils s'y prêtent. }\end{array}$ \\
\hline Transférabilité & 22 & $\begin{array}{l}\text { Explorer les répercussions de l'étude au vu } \\
\text { du potentiel d'application ou de transfert } \\
\text { des résultats. }\end{array}$ \\
\hline Prochaines étapes & 23 & $\begin{array}{l}\text { Proposer d'autres étapes ou domaines de } \\
\text { recherche à explorer. }\end{array}$ \\
\hline Conclusion & 24 & $\begin{array}{l}\text { Veiller à ce que la conclusion comprenne } \\
\text { les données et l'analyse et qu'elle réponde } \\
\text { à l'objectif de l'étude. }\end{array}$ \\
\hline
\end{tabular}

Abréviation : №, Nombre

\section{Références}

1. Ahmad R, Iwami M, Castro-Sánchez E, Husson F, Taiyari $\mathrm{K}$, Zingg W, Holmes A. Defining the user role in infection control. J Hosp Infect. 2016 Apr;92(4):321-7. doi: 10.1016/j. jhin.2015.09.018.

2. Lincoln YS, Guba EA. Naturalistic inquiry. Beverly Hills, CA: Sage; 1985.

3. Tong A, Sainsbury P, Craig J. Consolidated criteria for reporting qualitative research (COREQ): a 32-item checklist for interviews and focus groups. Int J Qual Health Care 2007 Dec;19(6):349-57

4. Mays N, Pope C. Qualitative research in health care. Assessing quality in qualitative research. BMJ. 2000 Jan $1 ; 320(7226): 50-2$.

5. Rowan M, Huston P. Qualitative research articles: Information for authors and peer reviewers. CMAJ 1997 Nov 15;157(10):1442-6.

6. The Equator Network (Enhancing the QUAlity and Transparency Of health Research). Oxford: EQUATOR Network; 2015. http://www.equator-network.org/.

7. Renseignements à l'intention des auteurs. Relevé des maladies transmissibles au Canada 2016;42:20-1. http:// www.phac-aspc.gc.ca/publicat/ccdr-rmtc/16vol42/drrm42-1/ar-04-fra.php. 